\title{
MULTIRRESISTENCIA Y FACTORES ASOCIADOS A LA PRESENCIA DE BETALACTAMASAS DE ESPECTRO EXTENDIDO EN CEPAS DE Escherichia coli PROVENIENTES DE UROCULTIVOS
}

\author{
Milagros N. Yábar ${ }^{1, a}$, Berenice Curi-Pesantes ${ }^{1, a}$, Carolina A. Torres ${ }^{1, a}$, Renzo Calderón-Anyosa ${ }^{1, a}$, \\ Maribel Riveros ${ }^{2, b}$, Theresa J. Ochoa ${ }^{1,2, c}$
}

\begin{abstract}
RESUMEN
El tratamiento empírico para la infección urinaria se ve complicado frente a la presencia de multirresistencia y de betalactamasas de espectro extendido (BLEE). El objetivo del estudio fue describir los patrones de resistencia antibiótica de cepas de Escherichia coli aisladas en urocultivos y los factores clínico-epidemiológicos asociados a la presencia de BLEE en un grupo pediátrico y adulto. Se recolectaron durante 14 meses, 353 cepas provenientes de Emergencia y Hospitalización del Hospital Cayetano Heredia, 45,9\% fueron cepas multirresistentes. La incidencia de BLEE en población pediátrica fue 16,3\% vs. $31,1 \%$ en la adulta, el $63,6 \%$ provenía de pacientes ambulatorios. La presencia de BLEE se asoció con encontrarse hospitalizado en pediatría, así cómo al uso de pañal y vejiga neurogénica en adultos. Estos factores deben considerarse al momento de elegir un tratamiento antibiótico. Asimismo, es necesario implementar programas de reporte epidemiológico y modelos de prevención de factores de riesgo.
\end{abstract}

Palabras clave: Escherichia coli; Infecciones urinarias; Farmacorresistencia múltiple; Resistencia a Medicamentos, Betalactamasas (fuente: DeCS BIREME)

\section{MULTIRESISTANCE AND FACTORS ASSOCIATED WITH THE PRESENCE OF EXTENDED-SPECTRUM BETA-LACTAMASES IN Escherichia coli STRAINS ISOLATED FROM URINE CULTURE}

\begin{abstract}
The empirical treatment of urinary infections is complicated by the presence of multiresistance and resistance to extendedspectrum beta-lactamases (ESBLs). The objective of this study was to describe the patterns of antibiotic resistance of Escherichia coli strains isolated from urine cultures and the clinical-epidemiological factors associated with the presence of ESBLs in a pediatric and an adult group. A total of 353 strains were collected from the Emergency and Hospitalization Sector of the Cayetano Heredia Hospital over 14 months, and $45.9 \%$ of the isolated strains were multiresistant. The rate of resistance to ESBLs in the pediatric and adult population was $16.3 \%$ and $31.1 \%$, respectively, and $63.6 \%$ of the resistant strains were isolated from outpatients. The presence of ESBLs was associated with hospitalization in pediatrics, use of diapers, and the presence of neurogenic bladder in adults. These factors should be considered in selection of an antibiotic treatment. Moreover, epidemiological reporting programs and models should be implemented for reduction of risk factors.
\end{abstract}

Keywords: Escherichia coli; Urinary tract infections; Multi-drug resistance; Antibiotic resistance; beta-Lactamases (source: MeSH NLM)

\section{INTRODUCCIÓN}

La infección del tracto urinario (ITU) es la segunda causa de enfermedad en pacientes ambulatorios y la segunda causa de infección intrahospitalaria en nuestro medio (1), siendo Escherichia coli el causante del $80-90 \%$ de casos ${ }^{(2)}$. El adecuado tratamiento antibiótico empírico requiere de reportes periódicos locales de sensibilidad antibiótica y protocolos de manejo aplicables al contexto geográfico y sociocultural del paciente. La resistencia antibiótica se atribuye, en parte, a la falta de programas de vigilancia y control epidemiológico, y al consecuente uso irracional e inapropiado de antibióticos, alcanzando este último, valores de hasta un $84 \%$ en zonas periurbanas en población pediátrica ${ }^{(3)}$. El estudio de sensibilidad antibiótica es útil para la toma de decisiones terapéuticas, el adecuado

\footnotetext{
Facultad de Medicina Alberto Hurtado, Universidad Peruana Cayetano Heredia. Lima, Perú.

Instituto de Medicina Tropical "Alexander von Humboldt", Universidad Peruana Cayetano Heredia, Lima, Perú

Médico cirujano; ${ }^{\mathrm{b}}$ bióloga; ${ }^{\mathrm{c}}$ médico infectólogo pediatra

Recibido: 05/06/2017 Aprobado: 08/11/2017 En línea: 01/12/2017
}

Citar como: Yábar MN, Curi-Pesantes B, Torres CA, Calderón-Anyosa R, Riveros M, Ochoa TJ. Multirresistencia y factores asociados a la presencia de betalactamasas de espectro extendido en cepas de Escherichia coli provenientes de urocultivos. Rev Peru Med Exp Salud Publica. 2017;34(4):660-5. doi: 10.17843/rpmesp.2017.344.2922 
manejo de la infección y la prevención de morbimortalidad en los pacientes.

Los betalactámicos se encuentran entre los antibióticos más utilizados en el tratamiento de este tipo de infecciones a nivel local ${ }^{(4)}$. No obstante, la detección de resistencia a estos agentes antimicrobianos, a menudo mediada por betalactamasas de espectro extendido (BLEE), ha ido en aumento a nivel mundial en los últimos años, dificultando el tratamiento. Es de gran importancia el aumento en la detección de BLEE en infecciones urinarias adquiridas en la comunidad (ITU-AC), lo cual requiere el estudio de factores de riesgo específicos a esta población. Hace más de 10 años, la prevalencia reportada para cepas portadoras de BLEE adquiridas en la comunidad era menor al $5 \%{ }^{(5)}$, mientras que en el 2015 se reportó en Chile un valor cercano al $17 \%{ }^{(6)}$.

El objetivo de este estudio fue describir los patrones de resistencia antibiótica y la incidencia del fenotipo BLEE en cepas de E. coli aisladas de urocultivos de pacientes del Hospital Cayetano Heredia $(\mathrm{HCH})$; asimismo, determinar los factores clínicos y epidemiológicos relacionados a la presencia de BLEE.

\section{EL ESTUDIO}

Estudio transversal realizado durante noviembre de 2012 a diciembre de 2013 en el Hospital Cayetano Heredia $(\mathrm{HCH})$, el cual es un hospital de referencia del tercer nivel para la zona norte de Lima que atiende a una población aproximada de 3 millones de personas ${ }^{(7)}$. Se recolectaron cepas de $E$. coli de urocultivos de pacientes pediátricos y adultos provenientes de los servicios de Emergencia y Hospitalización. Se excluyeron las muestras de aquellos pacientes que contaban con un urocultivo positivo previo, procesado en el laboratorio del $\mathrm{HCH}$ en un tiempo menor de dos semanas, por la posibilidad de corresponder al mismo patógeno y, por ende, sobrestimar dicho patrón de resistencia.

Los resultados del urocultivo y antibiograma fueron tomados de los registros del Laboratorio Central del $\mathrm{HCH}$, donde se emplea la técnica de difusión de disco de Kirby-Bauer bajo los lineamientos de las guías del Clinical Laboratory Standards Institute (CLSI) ${ }^{(8)}$. Los antibióticos empleados fueron: amikacina, amoxicilina-ácido clavulánico, ampicilina, ampicilina-sulbactam, aztreonam, cefalexina, cefotaxima, ceftazidime, ceftriaxona, ciprofloxacino, trimetropin-sulfametoxazol, gentamicina, imipenem, meropenem, nitrofurantoína, y norfloxacino. Los datos clínico epidemiológicos fueron recolectados de las historias clínicas.

El protocolo del estudio fue aprobado por el comité de ética del hospital, previo a la recolección de datos. No se requirió consentimiento informado debido a que solo se

\section{MENSAJES CLAVE}

Motivación para realizar el estudio. Resaltar la situación epidemiológica de las infecciones urinarias por Escherichia coli en nuestro medio y los patrones de resistencia que puedan servir de guías para el tratamiento empírico.

Principales hallazgos. De las 353 cepas analizadas del Hospital Cayetano Heredia, el $45,9 \%$ resultó ser multirresistente. La incidencia de BLEE en población pediátrica fue 16,3 y $31,1 \%$ en la adulta. Se asoció con encontrarse hospitalizado en pediatría, y con el uso de pañal y vejiga neurogénica en adultos.

Implicancias. Tras encontrar altas tasas de resistencia antibiótica, hacemos un llamado de salud pública frente al uso indiscriminado e inadecuado de antibióticos, de manera que se establezcan programas de vigilancia que permitan prevenir la emergencia y diseminación de gérmenes resistentes.

revisaron las historias clínicas y se analizaron las cepas aisladas, prescindiendo de identificadores personales. La determinación de la presencia del fenotipo BLEE se hizo en el Laboratorio de Enfermedades Entéricas, Nutrición y Resistencia Antimicrobiana (LEEN-RA) del Instituto de Medicina Tropical de la Universidad Peruana Cayetano Heredia (UPCH), mediante el método de sinergia de doble disco ${ }^{(8)}$.

Se definió al paciente adulto como aquel mayor o igual a 14 años; ITU severa fue aquella que se acompaña de sepsis, oliguria/anuria, globo vesical o elevación de la creatinina plasmática; ITU intrahospitalaria fue aquella que se presenta luego de 48 horas de la hospitalización, sin evidencia de infección al ingreso o en paciente con antecedente de haber sido hospitalizado en el último mes; multirresistencia se definió como resistencia a, al menos, un antibiótico de tres o más categorías de antibióticos, determinadas por la Sociedad Europea de Microbiología Clínica y Enfermedades Infecciosas ${ }^{(9)}$.

Se realizó un análisis bivariado estableciendo las características demográficas y clínicas relacionadas a resistencia antibiótica. Se analizaron un total de 19 factores de riesgo para infección urinaria. Las variables utilizadas fueron las siguientes: edad, sexo, procedencia (hospitalización vs. emergencia), diabetes mellitus, inmunodeficiencia, uso de pañal, reflujo vesicouretral, presencia de malformaciones genitourinarias, trasplante renal, hiperplasia benigna de próstata, vejiga neurogénica, uso de catéter urinario, incontinencia urinaria, litiasis renal, infección intrahospitalaria, uso de antibiótico en los últimos tres meses, ITU en el último año e ITU severa. Se calcularon las razones de prevalencia (RP) con intervalos de confianza al 95\% (IC95\%) mediante el modelo generalizado lineal, con familia binomial y link log para aquellas características asociadas a la presencia del fenotipo BLEE. Un valor de $p<0,05$ fue considerado estadísticamente significativo. Se utilizó el entorno de software libre $\mathrm{R}$. 


\section{HALLAZGOS}

Se incluyeron 353 cepas de E. coli, el 82,7\% (292/353) de cepas provinieron de muestras de pacientes adultos, el $78,7 \%(278 / 353)$ de sexo femenino y el $73,3 \%$ (259/353) proveniente de Emergencia. La resistencia antibiótica encontrada en el total de la muestra fue mayor para trimetropin-sulfametoxazol $(88,9 \%, 314 / 353)$, ampicilina $(83,5 \%, 295 / 353)$ y cefotaxima $(76,0 \%, 146 / 192)$. La resistencia a ciprofloxacino, cefalexina y ceftriaxona fueron $58,1 \%$ (204/351), 53,8\% (56/104) y 43,7\% (154/352), respectivamente. Amikacina $(4,5 \%, 16 / 353)$ y nitrofurantoína $(3,3 \%, 12 / 353)$ mostraron las frecuencias más bajas de resistencia en la población estudiada (Figura 1). No se halló resistencia elevada a imipenem o meropenem, sin embargo $16,8 \%(17 / 101)$ de cepas mostraron resistencia antibiótica intermedia a estos fármacos. La recolección de datos asociados a la infección mostró que 72 de 146 pacientes tenían una infección intrahospitalaria, de las cuales el $77,7 \%$ (56/72) fueron infecciones severas. El grupo pediátrico presentó tasas de resistencia menores que el grupo adulto para ciprofloxacino $(p<0,001)$, ceftazidima y cefotaxima $(p<0,001)$, ceftriaxona y cefalexina $(p<0,05)$, así como a amoxicilina-ácido clavulánico y ampicilina-sulbactam $(p<0,05)$ (Figura 1).

El $45,9 \%(162 / 353)$ de cepas fueron catalogadas como multirresistentes. En el análisis de patrones de resistencia antibiótica se incluyeron las cepas que fueron analizadas para el total de antibióticos incluidos en el antibiograma $(n=167)$. Al estudiar los patrones de resistencia, se evidenció que el $28,1 \%(47 / 167)$ de cepas eran resistentes a siete diferentes categorías de antibióticos de manera simultánea (trimetropinsulfametoxazol [SXT]; ampicilina [AMP]; ciprofloxacino y norfloxacino [CIP, NOR]; ceftriaxona, cefotaxima, ceftazidima
[CRO, CTX, CAZ]; aztreonam [ATM]; ampicilina-sulbactam y amoxicilina-ácido clavulánico [SAM, AMC]; gentamicina y amikacina [GEN, AMK]) (Tabla 1).

Se encontró el fenotipo BLEE en el 28,6\% (101/353) de la muestra, el 63,3\% (64/101) provino de pacientes en el servicio de Emergencias. La resistencia a amikacina en este grupo fue de $15,8 \%$ (16/101). Para la mayoría de estos pacientes no se lograron obtener datos completos respecto al tipo de infección, sin embargo, de las 37 cepas provenientes de pacientes hospitalizados, se confirmó que 26 se trataban de infecciones severas.

La frecuencia del fenotipo BLEE en población pediátrica fue de $16,3 \%(10 / 61)$ vs. $31,1 \%(91 / 292)$ en población adulta $(p<0,05)$. De modo exploratorio, se analizaron los factores relacionados a la presencia de BLEE, realizando un análisis estratificado por grupo etario y encontrándose una asociación en el grupo pediátrico con encontrarse hospitalizado (RP: 7,61 IC 95\%: 1,77 - 32,69); en adultos se encontró que el uso de pañal (RP: 1,88 IC 95\%: 1,21 $2,91)$ y vejiga neurogénica (RP: 2,08 IC 95\%: 1,21 - 3,58) estuvieron asociados. Los pacientes adultos mostraron una mayor asociación frente a la presencia de BLEE, sin embargo, el análisis estratificado por edades no mostró diferencias significativas frente a los otros factores.

\section{DISCUSIÓN}

Los betalactámicos y las quinolonas son antibióticos de primera línea en el tratamiento de la ITU por E. colien muchos países, incluyendo las recomendaciones de la Sociedad Americana de Enfermedades Infecciosas (IDSA) ${ }^{(10)}$. Nuestro estudio halló frecuencia elevadas de resistencia antibiótica a ambos, similares a estudios locales en población adulta

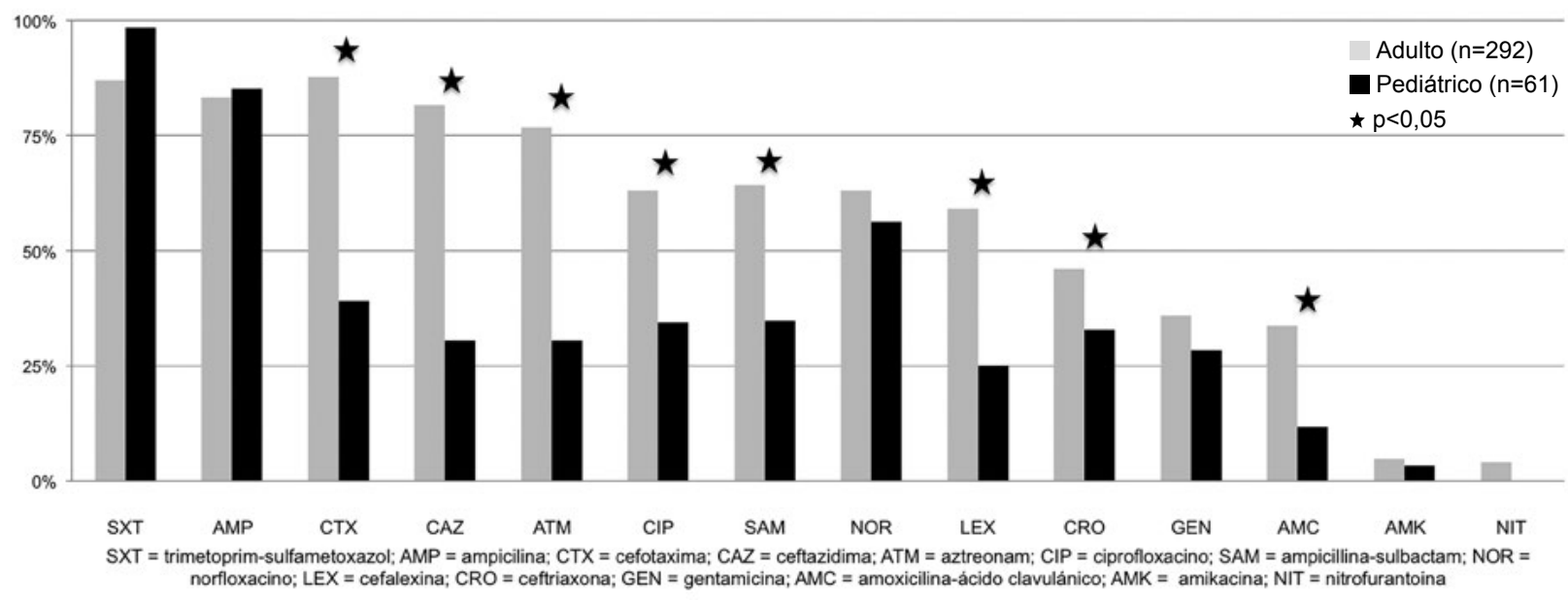

Figura 1. Resistencia antibiótica de E. coli aisladas en muestras de orina del Hospital Cayetano Heredia, según grupo etario. 
Tabla 1. Patrones de resistencia antibiótica de cepas de E. coli obtenidas de urocultivos del Hospital Cayetano Heredia.

\begin{tabular}{llr}
\hline N. ${ }^{\circ}$ de patrón & \multicolumn{1}{c}{ Patrones de resistencia antibiótica } & n/N (\%) \\
\hline I & SXT-AMP-CIP-CRO-ATM-CTX-CAZ-NOR-SAM-GEN-AMC & $13 / 167(7,8 \%)$ \\
II & SXT-AMP-CIP-CRO-ATM-CTX-CAZ-NOR-SAM-GEN-AMK & $3 / 167(1,8 \%)$ \\
III & SXT-AMP-CIP-CRO-ATM-CTX-CAZ-NOR-SAM-GEN & $20 / 167(11,9 \%)$ \\
IV & SXT-AMP-CIP-CRO-ATM-CTX-CAZ-NOR-SAM-AMC & $11 / 167(6,6 \%)$ \\
V & SXT-AMP-CIP-CRO-ATM-CTX-CAZ-NOR-GEN & $13 / 167(7,8 \%)$ \\
VI & SXT-AMP-CIP-CRO-ATM-CTX-CAZ-NOR-SAM & $10 / 167(6 \%)$ \\
VII & SXT-AMP-CIP-CRO-CTX-CAZ-NOR-SAM-AMC & $4 / 167(2,4 \%)$ \\
VIII & SXT-AMP-CIP-CRO-ATM-CTX-NOR-SAM-GEN & $3 / 167(1,8 \%)$ \\
IX & SXT-AMP-CIP-CRO-ATM-CTX-CAZ-NOR & $6 / 167(3,6 \%)$ \\
X & SXT-AMP-CIP-NOR-GEN & $3 / 167(1,8 \%)$ \\
XI & SXT-AMP-CIP-NOR & $6 / 167(3,6 \%)$ \\
Resistencia a dos antibióticos & $11 / 167(6,6 \%)$ \\
Resistencia a un antibiótico & $6 / 167(3,6 \%)$ \\
Pansensible & $1 / 167(0,6 \%)$ \\
Otros patrones & & $56 / 167(33,5 \%)$ \\
Total & $167(100 \%)$ \\
\hline
\end{tabular}

SXT = trimetoprim-sulfametoxazol; $\mathrm{AMP}=$ ampicilina; $\mathrm{CTX}=$ cefotaxima; $\mathrm{CAZ}=$ ceftazidima; $\mathrm{ATM}=$ aztreonam; CIP = ciprofloxacino; $\mathrm{SAM}=$ ampicillinasulbactam; NOR = norfloxacino; $\mathrm{LEX}=$ cefalexina; $\mathrm{CRO}$ = ceftriaxona; GEN = gentamicina; $\mathrm{AMC}$ = amoxicilina-ácido clavulánico; $\mathrm{AMK}$ = amikacina; NIT = nitrofurantoína

[ampicilina (85,7\%) y ciprofloxacino $(65,0 \%)]^{(11)}$ y pediátrica (ciprofloxacino 19,2\%) ${ }^{(12)}$. Creemos que las menores frecuencias de resistencia en la población pediátrica, pueden atribuirse a una menor frecuencia de enfermedades crónicas, institucionalización, inmunosupresión y tratamiento antibiótico previo, asociados al desarrollo de resistencia antibiótica. Se encontró $50 \%$ de resistencia a la cefalexina y ceftriaxona, sin embargo, estos antibióticos continúan siendo empleados como tratamiento de primera línea según las guías locales ${ }^{(4)}$. Únicamente seis de los 16 antibióticos analizados mostraron frecuencia de resistencia inferiores al 35\% (AMC, AMK, GEN, NIT y carbapenems).

Los aminoglicósidos aún mantienen tasas de resistencia baja en nuestro contexto local (aproximadamente $8 \%$ para el primer trimestre del 2015) ${ }^{(7)}$, por lo que deben ser considerados como tratamiento de primera línea en el paciente hospitalizado. La nitrofurantoína se encuentran indicada como terapia de primera línea para la ITU no complicada o cistitis en pacientes adultos y en niños mayores de un mes, además ha demostrado ser costo-efectiva ${ }^{(13)}$.

Estudios de multirresistencia en la región de las Américas reportan hasta un $95 \%$ de resistencia en cepas $E$. coli uropatógenas a, al menos, un antibiótico y $42 \%$ de multirresistencia ${ }^{(14)}$. Este dato en nuestro estudio podría estar subestimado, debido a que no fueron analizadas todas las categorías de antibióticos necesarias para definir dicho término en el total de la muestra. Los codificadores de betalactamasas (TEM, SHV, CTX-M) suelen asociarse a genes de resistencia a otros grupos de antibióticos, generando bacterias multirresistentes, principalmente hacia aminoglicósidos y quinolonas ${ }^{(15)}$, y aunque estas distinciones moleculares no se han analizado en nuestra investigación, revisando el patrón de resistencia, se podría inferir el tipo de mecanismo de resistencia que produce. Un estudio previo sugiere que la presencia de integrones 1 y 2 han demostrado tener una relación directa con los genes de resistencia hacia distintas familias de antibióticos ${ }^{(16)}$.

Este estudio sugiere que el uso de pañal y la vejiga neurogénica son factores de riesgo para la infección por E. coli productora de BLEE en pacientes mayores de 14 años. Un estudio local identificó, además, una asociación con el uso previo de antibióticos y el antecedente de hospitalización y cirugía previa para el desarrollo de una ITU-AC por bacterias productoras de BLEE (17). Por otro lado, un estudio colombiano halló en 2124 muestras, que el diagnóstico de ITU complicada (pielonefritis, malformación genitourinaria, inmunodeficiencia, infección en hombres o gestación) fue el único factor asociado a la ITU-AC por $E$. coli productora de BLEE ${ }^{(18)}$.

En la muestra pediátrica, el único factor que mostró asociación con la infección por cepas productoras de BLEE fue encontrarse hospitalizado; sin embargo, la prevalencia de BLEE en pediatría aún es baja y es probable que el tamaño de la muestra haya limitado el análisis de factores de riesgo. El porcentaje de resistencia intermedia a carbapenems hallado en este estudio debe ser monitorizado de manera periódica. Este dato requiere ser manejado de manera individual y en conjunto con el resto de datos dentro del antibiograma.

En este trabajo se presenta una muestra amplia de pacientes ambulatorios y hospitalizados de un hospital de Perú, incluyendo pacientes pediátricos y adultos, a diferencia de la mayoría de estudios que suelen presentar un solo grupo etario y pone en evidencia las elevadas frecuencias de resistencia que se presentan en nuestro 
medio. Somos conscientes de que existe un sesgo de selección que nos restringe de realizar inferencias a nivel nacional, sin embargo, sugerimos la implementación de estudios periódicos de mayor magnitud que permitan seguir la tendencia local y regional. El estudio de patrones de resistencia antibiótica es una herramienta útil para guiar el tratamiento empírico de una infección, así como demostrar la importancia de realizar el estudio de antibiograma ante la sospecha de una infección.

Al encontrarse frente a una cepa productora de BLEE debemos considerar las características propias de la infección, los factores asociados a esta y los reportes locales de resistencia. Cabe mencionar que el acceso a antibióticos sin prescripción médica en Perú es también un factor mayor, que contribuye al desarrollo de resistencia antibiótica; para lo cual será necesario implementar programas integrales de vigilancia y control epidemiológico, con la participación informada del profesional de salud. El aumento de la resistencia antibiótica en el ámbito comunitario es un problema de salud pública que requiere mayores estudios. Actualmente se encuentra en investigación el rol epidemiológico de cepas comensales de $E$. coli como reservorios de genes productores de betalactamasas ${ }^{(19)}$. Creemos necesario implementar programas de prevención de infecciones, así como de identificación de factores de riesgo en la población, logrando priorizar el tratamiento empírico en pacientes de alto riesgo, con el objetivo de disminuir la transmisión y reducir los costos en el manejo del paciente infectado por bacterias multirresistentes ${ }^{(20)}$.

Contribuciones de autoría: MNY, BCP, CAT y TJO participaron en la concepción, diseño del artículo, redacción inicial del manuscrito y la obtención de financiamiento. MNY, BCP y CAT participaron en el enrolamiento de sujetos y recolección de resultados, junto a RCA y MRR realizaron el análisis e interpretación de datos y redacción del artículo. Todos los autores revisaron y aprobaron la versión final del manuscrito.

Financiamiento: estudio financiado por la Beca Anual "Francisco Tejada y Semíramis Reátegui” del año 2012, otorgado por la facultad de Medicina Alberto Hurtado de la Universidad Peruana Cayetano Heredia a Milagros N. Yábar, Berenice Curi-Pesantes y Carolina A. Torres

Conflictos de interés: los autores declaran no tener conflictos de interés en la publicación de este artículo.

\section{REFERENCIAS BIBLIOGRÁFICAS}

1. Echevarría-Zarate J, Sarmiento Aguilar E, Osores-Plenge F. Infección del tracto urinario y manejo antibiótico. Acta méd peruana. 2006;23:126-31.

2. Ceballos O, Erazo I, López R, Fuentes R, Campillay C. Caracterización de Lactantes con infección del Tracto Urinario: 20092010: Hospital Regional de Antofagasta. ANACEM. 2010; 4(1):14-17.

3. Ecker L, Olarte L, Vilchez G, Ochoa TJ, Amemiya I, Gil AI, et al. Physicians' responsibility for antibiotic use in infants from periurban Lima, Peru. Rev Panam Salud Publica. 2011;30(6):574-9.

4. Ministerio de Salud. Guía de Práctica Clínica de Infección del Tracto Urinario (ITU) [Internet]. Lima: Ministerio de Salud; 2015 [citado el 10 may 2017]. Disponible en: http://www. hospitalcayetano.gob.pe/transparencia/ images/stories/resoluciones/RD/ RD2015/rd_104_2015.pdf

5. Calbo E, Romaní V, Xercavins M, Gómez L, Vidal CG, Quintana S, et al. Risk factors for community-onset urinary tract infections due to Escherichia coli harbouring extended-spectrum betalactamases. J Antimicrob Chemother. 2006;57(4):780-3.

6. Avilés C, Betancour P, Velasco CL, Godoy R, BarthelE, Martínez F. Factores asociados a infecciones urinarias producidas por enterobacterias productoras de betalactamasas de espectro extendido: una cohorte prospectiva. Rev Chilena Infectol. 2016;33(6):628-634. doi: 10.4067/ S0716-10182016000600004.

7. Ministerio de Salud. Análisis Situacional de Salud del Hospital Nacional Cayetano Heredia 2015 [Internet]. Lima: Oficina de Epidemiología y Salud Ambiental. Hospital Nacional Cayetano; 2015. Disponible en: http://www.hospitalcayetano.gob.pe/ Inicio/images/Documentos/Epidemio/ ANALISISSITUACIONALDESALUDHCH2015.pdf

8. Clinical and Laboratory Standards Institute (CLSI). Performance Standards for Antimicrobial Susceptibility Testing; Twenty-Third Informational Supplement. CLSI document M100-S23. Wayne, PA: Clinical and Laboratory Standards Institute; 2013.

9. Magiorakos AP, Srinivasan A, Carey RB, Carmeli Y, Falagas ME, Giske CG, et al. Multidrug-resistant, extensively drug-resistant and pandrug-resistant bacteria: an international expert proposal for interim standard definitions for acquired resistance. Clin Microbiol Infect. 2012;18(3):268-81. doi: 10.1111/j.14690691.2011.03570.x.
10. Gupta K, Hooton TM, Naber KG, Wullt B, Colgan R, Miller LG, et al. International Clinical Practice Guidelines for the Treatment of Acute Uncomplicated Cystitis and Pyelonephritis in Women: A 2010 Update by the Infectious Diseases Society of America and the European Society for Microbiology and Infectious Diseases. Clin Infect Dis. 2011;52(5):e103-20. doi: 10.1093/cid/ ciq257.

11. Gonzales Camarena DE, Jaulis Solorzano JF, Tapia Egoavil EZ, Samalvides Cuba F. Sensibilidad antibiótica de bacterias causantes de infecciones del tracto urinario en un hospital general: Enero - junio del año 2008. Rev Med Hered. 2009;20:1115.

12. Llerena Torres J, López Apac L, López Huayamares M, Lucana Masías $M$. Etiología y sensibilidad antibiótica de infección del tracto urinario en menores de 4 años en el Instituto Nacional de Salud del Niño durante el año 2008. [Tesis para obtener el grado de especialista]. Lima: Instituto Nacional de Salud del Niño; 2011.

13. McKinnell JA, Stollenwerk NS, Jung CW, Miller LG. Nitrofurantoin Compares Favorably to Recommended Agents as Empirical Treatment of Uncomplicated Urinary Tract Infections in a Decision 
and Cost Analysis. Mayo Clin Proc. 2011;86(6):480-8. doi: 10.4065/ mcp.2010.0800.

14. Oliveira FA, Paludo KS, Arend LN, Farah SM, Pedrosa FO, Souza EM, et al. Virulence characteristics and antimicrobial susceptibility of uropathogenic Escherichia coli strains. Genet Mol Res. 2011;10(4):4114-25. doi: 10.4238/2011. October.31.5.

15. Mosquito S, Ruiz J, Bauer JL, Ochoa TJ. Mecanismos moleculares de resistencia antibiótica en Escherichia coli asociados a Diarrea. Rev Peru Med Exp Salud Pública. 2011;28(4):648-56.

16. Rodas $C$, Halvorsen $K$, Iñiguez $V$. Multiresistencia antimicrobiana asociada a integrones en enteropatógenos de la diarrea infantil y Escherichia coli de la flora normal en niños menores de 5 años en la ciudad de La Paz. Cuad Hosp Clín. 2005;50(2):38-48

17. Castillo-Tokumori F, Irey-Salgado C, Malaga G. Worrisome high frequency of extended-spectrum beta-lactamase-producing Escherichia coli in community-acquired urinary tract infections: a case-control study. Int J Infect Dis. 2017;55:16-19. doi: 10.1016/j.jid.2016.12.007.

18. Blanco VM, Maya JJ, Correa A, Perenguez M, Muñoz JS, Motoa G, et al. Prevalencia y factores de riesgo para infecciones del tracto urinario de inicio en la comunidad causadas por Escherichia coli productor de betalactamasas de espectro extendido en Colombia. Enferm Infecc Microbiol Clin. 2016;34(9):559-565. doi: 10.1016/j. eimc.2015.11.017.

19. Riveros Ramirez M, Marcelo M, Alcedo $\mathrm{K}$, Alejos S, Hinostroza N, Ruiz J, et al. De- tection of ESBL-producing E. coli isolates among healthy children in Peru. European Congress of Clinical Microbiology and Infectious Diseases; 2017 Apr 22; Vienna, Austria.

20. Tansarli GS, Karageorgopoulos DE, Kapaskelis A, Falagas ME. Impact of antimicrobial multidrug resistance on inpatient care cost: an evaluation of the evidence. $\mathrm{Ex}$ pert Rev Anti Infect Ther. 2013;11(3):32131. doi: 10.1586/eri.13.4.

Correspondencia: Carolina Alejandra Torres Pérez-Iglesias

Dirección: Av. Honorio Delgado 430, San Martín de Porras, Lima 33, Perú.

Teléfono: (+51) 3190000 ext 2715

Correo electrónico: caro.torrespi@gmail.com

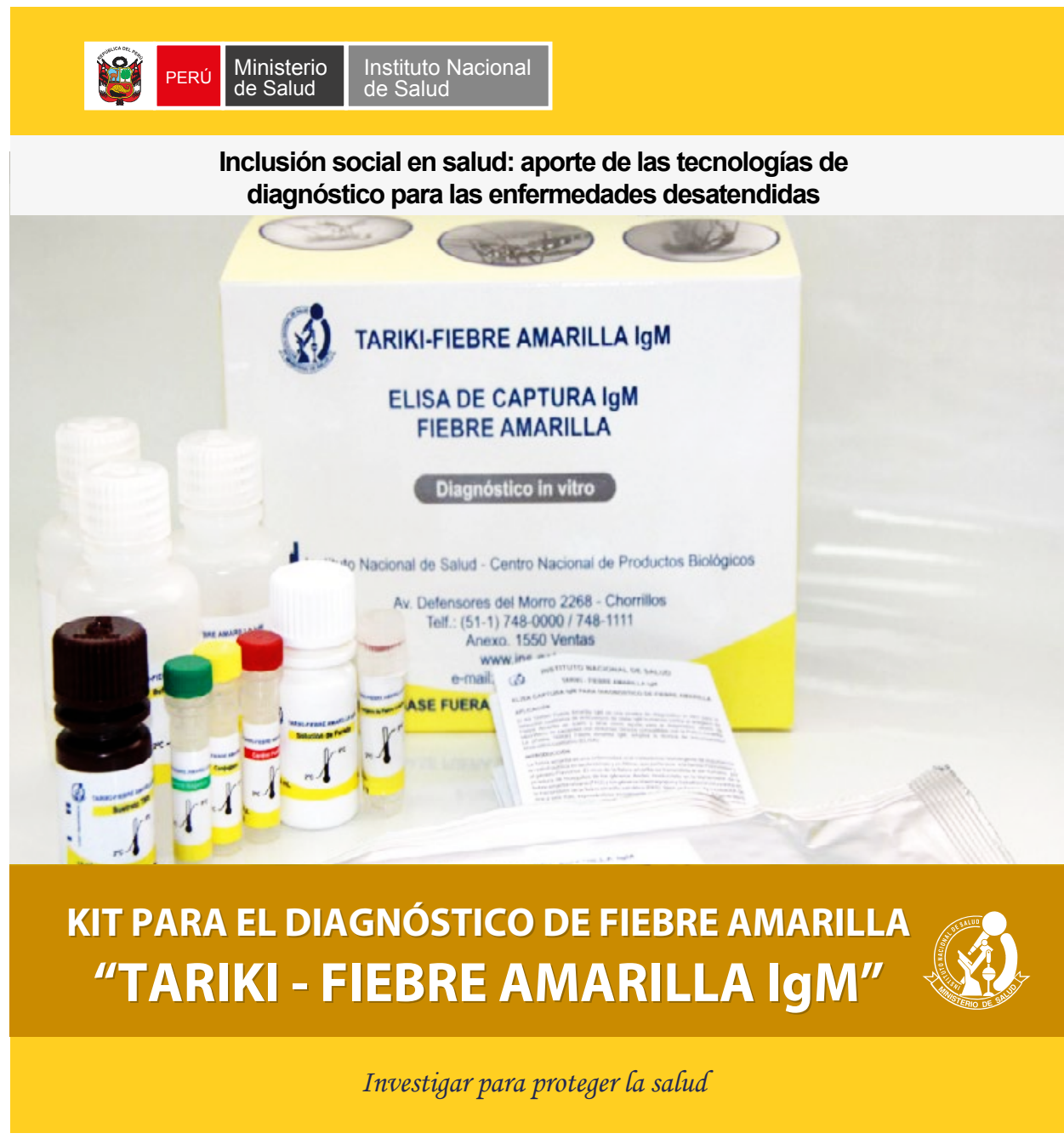

